2019-04

\title{
Characterising the deterioration of different plastics in air and seawater
}

\author{
Biber, NFA
}

http://hdl.handle.net/10026.1/13494

10.1016/j.marpolbul.2019.02.068

Marine Pollution Bulletin

Elsevier

All content in PEARL is protected by copyright law. Author manuscripts are made available in accordance with publisher policies. Please cite only the published version using the details provided on the item record or document. In the absence of an open licence (e.g. Creative Commons), permissions for further reuse of content should be sought from the publisher or author. 


\title{
Characterising the deterioration of different plastics in air and seawater
}

\author{
Nicolas F.A. Biber ${ }^{\mathrm{a}, *}$, Andy Foggo ${ }^{\mathrm{a}}$, Richard C. Thompson ${ }^{\mathrm{a}}$ \\ ${ }^{a}$ Marine Biology and Ecology Research Centre, School of Biological and Marine Sciences, \\ University of Plymouth, Drake Circus, Plymouth, PL4 8AA, United Kingdom
}

\begin{abstract}
In situ studies of plastic deterioration can help us understand the longevity of macroplastic as well as the generation of microplastics in the environment. Photo-oxidation contributing to the generation of microplastics in the marine environment was explored using four types of plastic (polyethene, polystyrene, poly(ethylene terephthalate) and Biothene $\AA$ exposed in light and in shade, in both air and sea water. Metrics for deterioration were tensile extensibility and oxidation rate. Measurements were conducted at intervals between 7 and 600 days' exposure. Deterioration was faster in air than in sea water and was further accelerated in direct light compared to shade. Extensibility and oxidation were significantly inversely correlated in samples exposed in air. Samples in sea water lost extensibility at a slower rate. Polystyrene, which enters the waste stream rapidly due to its wide application in packaging, deteriorated fastest and is, therefore, likely to form microplastics more rapidly than other materials, especially when exposed to high levels of irradiation, for example when stranded on the shore.
\end{abstract}

Keywords: plastic deterioration, natural environment, polystyrene, photo-oxidation, tensile properties, FTIR

\section{Introduction}

This study characterises molecular and mechanical aspects of deterioration of polyethene, polystyrene, poly(ethylene-terephthalate) and Biothene®, an oxy-degradable polyethene advertised as "100\% degradable" , when exposed in air and sea water. The experiment was conducted in the natural

\footnotetext{
${ }^{*}$ Corresponding author

Email addresses: nicolas.biber@gmail.com (Nicolas F.A. Biber), a.foggo@plymouth.ac.uk (Andy Foggo), r.c.thompson@plymouth.ac.uk (Richard C. Thompson)
} 
environment to better inform predictions of the deterioration of marine debris.

Almost $40 \%$ of the demand for plastics in Europe comes from packaging (PlasticsEurope, 2012). Packaging applications dictate that the service lives of these products are very short, and that these materials enter the waste stream at a higher rate than plastics from other applications. One of the key attributes of plastic items is their durability; nonetheless, they will deteriorate in the environment over time, primarily due to exposure to UV radiation and mechanical abrasion (Colton et al., 1974; Gregory, 1978; Andrady, 2003; Thompson et al., 2004). Environmental contamination associated with plastic deterioration has been a concern since the 1970s (Colton et al., 1974; Gregory, 1978); more recently attention has focused upon microplastics (Thompson et al., 2004) and their environmental impacts, particularly in the marine environment (Teuten et al., 2007; Browne et al., 2008; van Cauwenberghe et al., 2012; Goldstein and Goodwin, 2013; Cole et al., 2014; Lee et al., 2014). Microplastics can be generated (among other sources) from the deterioration of larger plastic debris. Their presence in the ocean may often be traced back to deterioration of larger items, which have formed as a consequence of loss of structural integrity.

Marine debris is frequently washed ashore from the ocean but may previously have been dispersed at sea for extended periods of time. This is evidenced by remote beaches with no anthropogenic activity being littered with large quantities of plastics (Lucas, 1992; Barnes, 2005; Bond et al., 2014). Therefore, 'marine' debris may spend significant amounts of time on land, and may thus alternately be exposed to two environments that would affect deterioration in different ways. In order to further our understanding of the sources of variation in the deterioration of macroplastics and the generation of marine microplastics, it is valuable to compare the deterioration of plastics on land to that in seawater.

One significant contributory factor affecting plastic deterioration is the breaking of polymer chains at the molecular level (Klemchuck, 1990). Crosslinks of long polymer chains provide the material's cohesion in many types of plastic; the lower the molecular weight, the less mechanically stable the material is (Sawai et al., 2006). Light, especially UV radiation, causes polymer chains to break (Allen et al., 1983; Ranby, 1989; Gardette et al., 1995) in a reaction called photo-oxidation, which has been cited as the most important environmental cause of polymer deterioration (Carrasco et al., 2001). For example polyethene and polypropylene lose extensibility, mechanical integrity and molecular weight when exposed to UV radiation (Hamid and Prichard, 1991; Andrady et al., 1993a; Marek et al., 2006; Obadal et al., 2006; Singh and Sharma, 2008). Therefore, plastic deterioration can be quantified by 
assessing the material's tensile properties (ISO/DIS, 2009). Deterioration of plastics due to factors such as radiation or heat has been studied in laboratory settings (Israeli et al., 1994; Gardette et al., 1995; Nagai et al., 1999; Copinet et al., 2004), but these studies have been conducted largely as quality assessments on material stability. In order to understand the generation of microplastics, it is essential to explore deterioration processes in an environmental setting.

Effects of environmental exposure on plastics' tensile properties have been studied in both air and (surface) sea water (Pegram and Andrady, 1989; Andrady et al., 1993b,c). The evolution of chemical bonds resulting from oxidation have also been observed in simulated natural conditions on plastic pellets (Brandon et al., 2016). Plastic films (as opposed to pellets) offer the possibility to record changes in both tensile properties and chemical bonds. Plastic films also represent post-consumer waste as opposed to raw material waste and thus a different source of marine debris to plastic pellets.

Studies that observed material deterioration to date have also mostly focused on surface debris. However, plastic debris become negatively buoyant when organisms colonise them and thus alternate between being at the surface and submerged (Song and Andrady, 1991). Marine debris also become submerged by wind mixing, and it is estimated that this factor alone causes $60 \%$ of debris to be submerged at any given time (Kukulka et al., 2012). It is therefore pertinent to consider deterioration of plastics that are not at the surface; this study addresses this by exposing samples in one metre of sea water.

Photo-oxidation is initiated by the cleaving of a hydrogen atom off a polymer in the presence of oxygen, the polymer forming a peroxide. The high reactivity of the peroxide propagates this reaction throughout the material. The reaction is terminated by an oxygen radical cleaving its own polymer and forming a methylene group at one end and a carbonyl group at the other end (Kuzina and Mikhailov, 2001). This increase of characteristic polymer chain end groups presents the opportunity to detect and measure plastic deterioration at a level that is undetectable from the perspective of mechanical strength or visible material integrity. Carbonyl and/or hydroxyl density can be detected by infrared or X-ray photoelectron spectroscopy as an indicator of chain-ends that are typical of photo-oxidation (Allen et al., 1983; Nakayama et al., 1996).

The present study characterised deterioration processes causing loss of mechanic stability of plastics. This was achieved by assessing material integrity and by quantifying the role of oxidation as measured in chain end density using FTIR spectroscopy. To examine the environmental factors leading to generation of microplastics in the marine environment, two factors: 
medium (air versus sea water) and illumination (light versus shade) were considered. Three materials, polyethene, polystyrene and poly(ethyleneterephthalate), were selected for their wide application in packaging. In addition, Biothene $\mathbb{R}$ was selected as a representative of the plethora of materials that carry the label 'bio' either for being plant-based or for being 'biodegradable', to compare its deterioration characteristics to the other three materials. Biothene $(\mathrm{B}$ is advertised to have enhanced deterioration properties due to a metal ion additive (http://www.biothene.co.uk, Apr. 29 2012).

\section{Methods}

\subsection{Materials}

Four plastic types were sourced as newly produced carrier bags or films and prepared as described below. The materials were cut into $5 \times 30 \mathrm{~cm}$ strips.

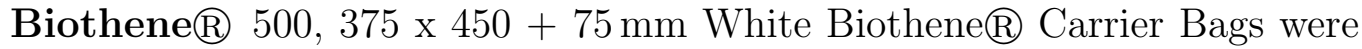
purchased from http://www.midpac.co.uk, 19 Binns Close, Tile Hill, Coventry, West Midlands, CV4 9TB, United Kingdom.

Polyethene 500, 375 x $450+75 \mathrm{~mm}$ White Polyethene Carrier Bags were purchased from http://www.midpac.co.uk, see address above.

Polystyrene One roll of $300 \mathrm{~mm}$ wide ST311050 Polystyrene Film $50 \mu \mathrm{m}$ thick was purchased from BANDO CO., LTD., 704-900, 358-66, Galsandong, Dalseo-gu, Daegu, 704-900 KOREA.

Poly(ethylene terephthalate) One roll of $300 \mathrm{~mm}$ wide Polymex PI700 matte translucent untreated polyester film $50 \mu \mathrm{m}$ thick was purchased from http://www.polymex.co.uk, Polymex Limited, Meridian House 62 Station Road, North Chingford, London, E4 7BA, United Kingdom.

To best address debris resulting from post-consumer plastics, commercial products were selected as test materials. The information available on these is limited (compared to raw materials), as some of it is protected as the manufacturers' intellectual property. Nevertheless, the materials could be characterised to a large extent. The volumetric mass densities of the materials were calculated from the sample area $(5 \times 30 \mathrm{~cm})$, thickness as measured 5 times per sample with a Vernier calliper accurate to $20 \mathrm{~m}$ and weight as measured with an analytical balance. Tensile extensibility and strength of each material were measured at time 0 . Densities and tensile properties are listed in Table 2.1. FTIR spectra of each material were also recorded at time 0 ; these spectra are illustrated in Figure 3. 
Table 1: Mean volumetric mass densities of 145 samples of each material type based on $5 \mathrm{x}$ $30 \mathrm{~cm}$ area, as well as thickness and mass of each sample; tensile strength and extensibility of 10 samples of Biothene(B)and poly(ethylene terephthalate) and 9 samples of polyethene.

\begin{tabular}{|c|c|c|c|c|c|}
\hline Material & $\begin{array}{l}\text { Density } \\
\left(\mathrm{g} \mathrm{cm}^{-3}\right)\end{array}$ & $\begin{array}{l}\text { Thickness } \\
(\mu \mathrm{m})\end{array}$ & Mass (mg) & $\begin{array}{l}\text { Tensile } \\
\text { strength } \\
\left(\mathrm{N} \mathrm{mm}^{-2}\right)\end{array}$ & $\begin{array}{l}\text { Tensile ex- } \\
\text { tensibility } \\
\text { (factor) }\end{array}$ \\
\hline \multirow[t]{2}{*}{ Biothene(R) } & $1.12 \pm 0.11$ & 43.17 & 715.17 & $6.39 \pm 0.95$ & 45.38 \\
\hline & & 4.94 & 23.95 & & 13.52 \\
\hline \multirow[t]{2}{*}{ Polyethene } & $1.05 \pm 0.1$ & $42.81 \pm 9.5$ & 662.83 & $7.03 \pm 0.85$ & 27.01 \\
\hline & & & 31.81 & & 3.68 \\
\hline \multirow[t]{2}{*}{ PET } & $1.15 \pm 0.03$ & $60 \pm 0.82$ & $1038.28 \pm$ & $0.80 \pm 0.14$ & 374.64 \\
\hline & & & 28.49 & & 28.74 \\
\hline \multirow[t]{2}{*}{ Polystyrene } & $1.1 \pm 0.19$ & 46.07 & 734.55 & $3.00 \pm 1.13$ & 53.52 \\
\hline & & 9.23 & 20.75 & & 12.28 \\
\hline
\end{tabular}

\subsection{Experimental design}

The experiment was laid out in an orthogonal design with 4 fixed factors: 'material', 'environment', 'lighting' and 'exposure duration'. Each level in the orthogonal combination of factors contained 5 replicates (five samples of the same material attached to each exposure rig). The experiment was set up in nine cohorts to be collected and analysed 7, 14, 21, 28, 100, 240, 360, 480 and 600 days after deployment. An initial deployment in which first sampling time was after 240 days revealed that substantial deterioration had already occurred in the first 240 days, especially in polystyrene, this prevented assessment of the deterioration indicators for some samples. Therefore, the 7 , 14, 21, 28 and 100 day cohorts were subsequently deployed to account for more rapid material changes or losses in select materials and treatments.

\subsection{Sample exposure}

Samples were exposed in sea water and air on 27th and 24th August 2010 respectively. In both environments one location illuminated by natural sunlight and one shaded location were selected; one set of samples was retained in constant dark conditions in the laboratory as a control (see Table 2.3). The light intensity in the illuminated location was 10 times stronger than in shade in air, and 5 times stronger than in shade in sea water. Light intensity in air was approximately 15 times that in sea water. No measurable light entered the control environment. Sea water salinity and temperature at sample depth at the exposure site were $29.5 \mathrm{ppt}$ and $11.8 \mathrm{C}$ respectively in May 2012. 
Table 2: Exposure locations in Plymouth, UK and corresponding means standard deviations of light intensities.

\begin{tabular}{lllll} 
Parameter & Sea water & Air & Control \\
\hline Site & Queen Anne's & Skardon Gar- & Plymouth Uni- \\
& Battery Marina, & den $\left(50.378^{\circ} \mathrm{N}\right.$, & versity, Labora- \\
& visitor pon- & $\left.4.137^{\circ} \mathrm{W}\right)$ & tory & \\
& toon $\left(50.365^{\circ} \mathrm{N}\right.$, & & \\
& $\left.4.132^{\circ} \mathrm{W}\right)$ in $1 \mathrm{~m}$ & & \\
& of water & & \\
Illuminated & $\begin{array}{l}\text { Along West side } \\
\text { of ponton, }\end{array}$ & South-facing & $\mathrm{n} / \mathrm{a}$ & \\
& wall, 3m above & & \\
Shaded & Underneath & $\begin{array}{l}\text { North-East fac- Hanging in a } \\
\text { pontoon }\end{array}$ & ing wall, 1.5 & cardboard box \\
& & to $2 \mathrm{~m}$ above & & \\
& & ground &
\end{tabular}

Wooden beam clamps were used to deploy the samples (O'Brine and Thompson, 2010). Each wooden beam clamp was designated to one treatment combination, bearing five samples of each material. Samples were cushioned with several layers of the same material in order to protect them from being torn off along the edge of the clamp. Staples were used to attach the samples and the two wooden beams forming the clamp were attached together using screws (Figure 2.3).

In sea water the clamps were weighted to prevent flotation and deployed at a depth of $1 \mathrm{~m}$. In air they were attached to a wall at the end of $30 \mathrm{~cm}$ long brackets that prevented the samples from being abraded against the wall.

In order to confirm and quantify the difference between illuminated and shaded conditions, relative light intensities were measured in both using a Skye Instruments PAR Quantum Sensor (SKP215).

\subsection{Measuring deterioration}

Prior to and after exposure, samples were collected and stored under controlled conditions and analysed as quickly as possible. They were weighed using an analytical balance, their thickness was measured in five random locations using a mechanical Vernier calliper accurate to $20 \mu \mathrm{m}$ and their surface area was assessed visually as a percentage of their original area.

\subsubsection{Tensile properties}

Extensibility (elongation at breaking point as a multiple of original length, 'strain') of one specimen per sample was assessed with an apparatus that 


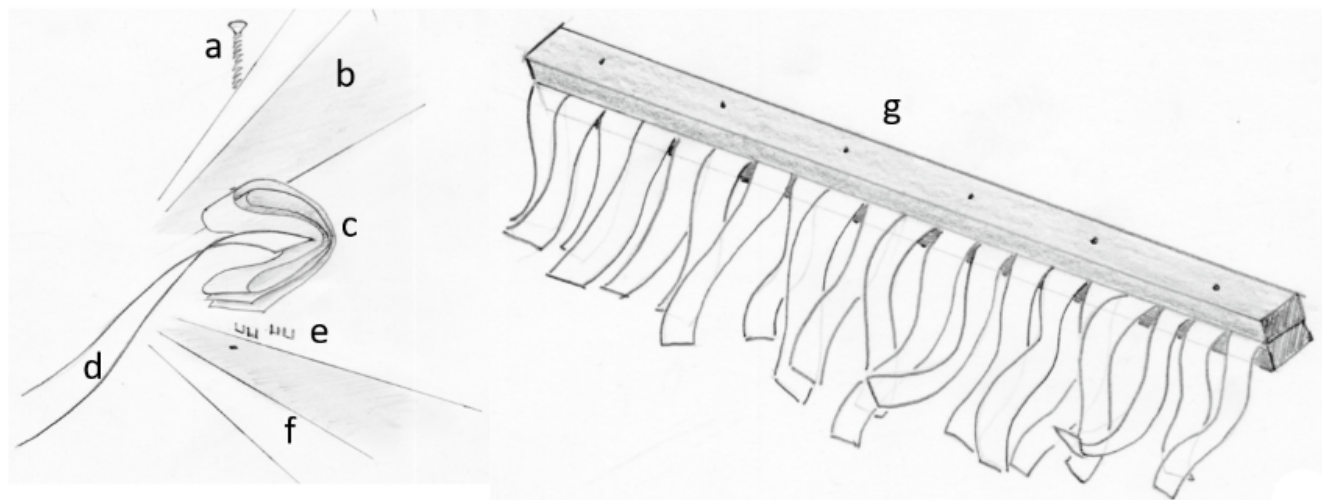

Figure 1: Detail of sample attachment method showing the brass screw (a), the upper beam (b), the cushioning material layers (c), the sample (d), the copper staples (e) and the lower beam (f) and wooden beam clamp with attached samples $(\mathrm{g})$.

complied with ISO 5893 (Instron, system ID $3345 \mathrm{k} 1669$, force transducer model 2519-104, capacity $500 \mathrm{~N}$ ). The elongation rate was set to $40 \mathrm{~mm} \mathrm{~min}^{-1}$. The specimens were cut into the shape illustrated in Figure 2.4.1 (British Standards Institution, 1996) using a Swann-Morton stainless steel surgical blade on a cutting mat. The specimen dimensions used were $b=10 \mathrm{~mm}$, $l_{3}=150 \mathrm{~mm}, l_{0}=50 \mathrm{~mm}$ and $l=50 \mathrm{~mm}(l$ was reduced according to British Standards Institution (1996) guidelines as a longer $l$ would cause specimen elongation to exceed instrument capacity); h was measured for each sample individually upon deployment.

\subsubsection{Fourier transform infrared spectroscopy (FTIR)}

Samples were cleaned of organic material using absolute ethanol prior to being processed. 5 replicate subsamples of each material were extracted from each sample from the proximal and the distal (relative to the point of attachment to the beam clamp) ends on both edges of the strip and from the centre of the strip. The replicates were placed on a two-part diamond window microscope slide and scanned 32 times using a Bruker Hyperion 1000 FTIR microscope operated with the OPUS TM 5.5 software package.

The resulting spectra of non-exposed material samples and samples exposed to illuminated air (exposed to the highest UV radiation) were examined visually to identify absorption bands that had changed over time. These bands were selected for statistical analysis (Figure 3). 


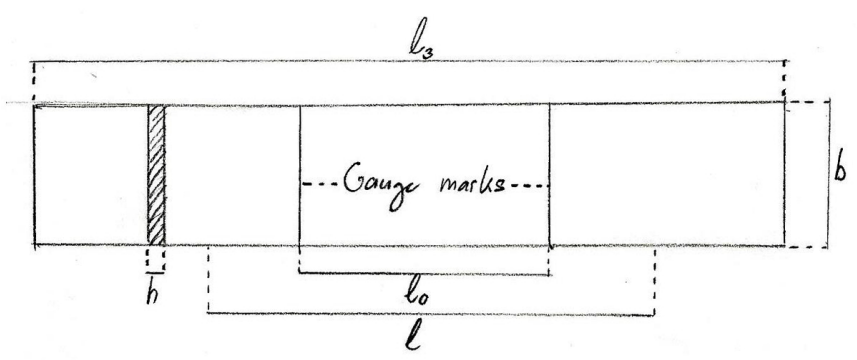

Figure 2: Figure 2: Specimen type 2 (British Standards Institution 1996) applied in the tensile properties test. $b$ : width, $h$ : thickness, $l$ : grip distance, $l_{0}$ : gauge length, $l_{3}$ :overall length.
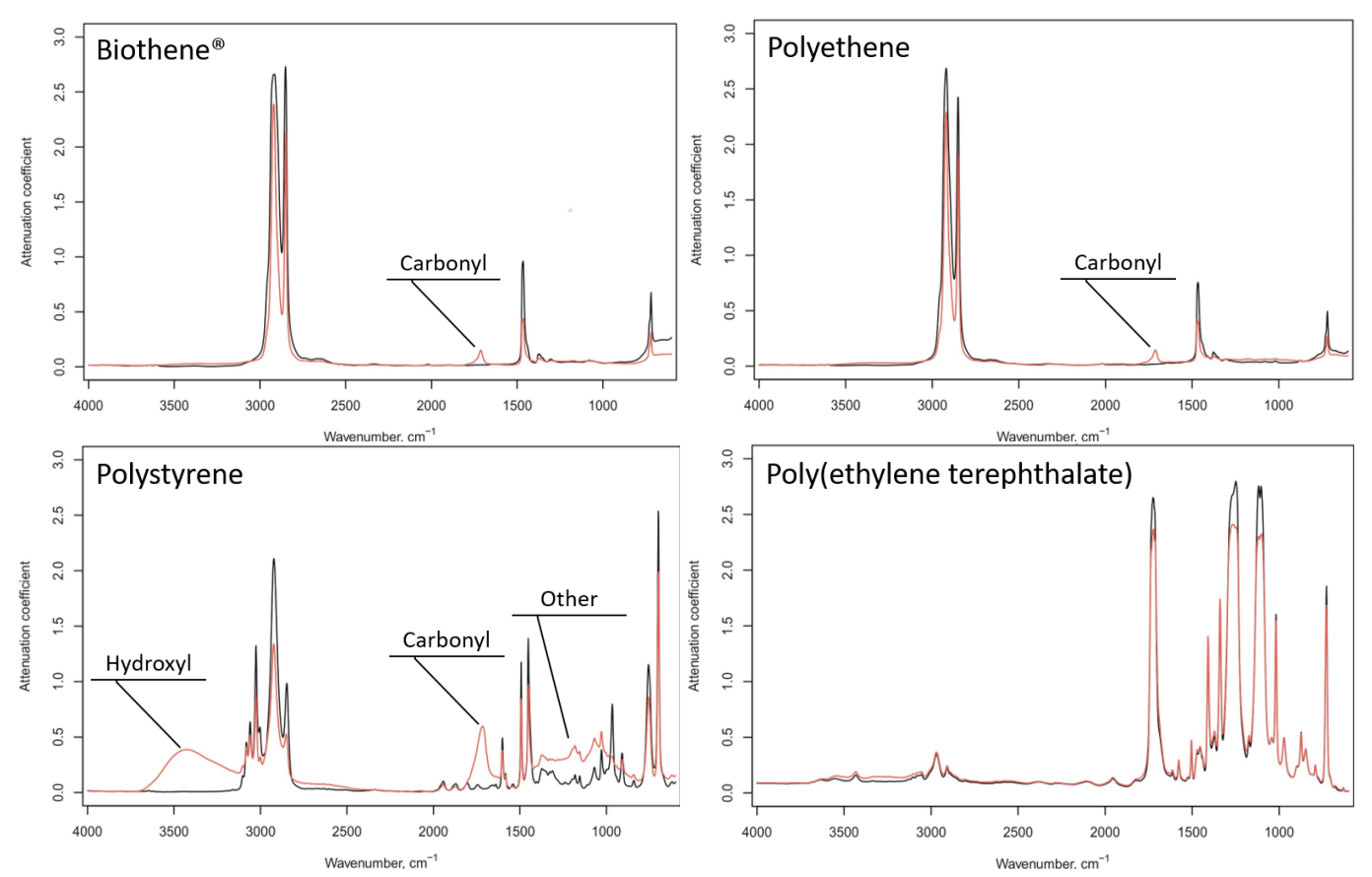

Figure 3: FTIR spectra (attenuation coefficient vs. wavenumber $\left(\mathrm{cm}^{-1}\right)$ ) of non-exposed (black solid line) and exposed in terrestrial light environment for 600 days (28 days for polystyrene) (red solid line). The peaks of the red vs. the black line were selected for further examination. No defined unique peaks could be identified for poly(ethylene terephthalate). 


\subsection{Data analysis}

Due to the large number of tested treatments, the sample size per treatment had to be kept small in order to keep the experiment manageable $(n=5)$. Therefore, the data analysis was designed to accommodate data with non-normally distributed residuals. PRIMER v6 (Clarke and Gorley, 2006) with the PERMANOVA+ add-in package (Anderson et al., 2008) perform univariate and multivariate analyses that are based upon triangular between-sample similarity or dissimilarity matrices calculated using a variety of metrics rendering analyses independent of the raw data error structure.

Deterioration of materials was quantified as the change of material properties over time. The significance of any differences between exposure times was tested using permutational multivariate analysis of variance (PERMANOVA).

The effect of light upon each material in each environment was tested using planned pair-wise contrasts in PERMANOVA, comparing samples that were exposed in well illuminated conditions with their counterparts in the shaded location. A significant difference between samples exposed in illuminated and shaded locations increasing along sampling dates would indicate that light influences material deterioration.

To test the relationship between oxidation and extensibility a PRIMER v6 RELATE test of correlation between extensibility and carbonyl absorption band value was conducted for each material separately using Spearman's Rho. A poor correlation would indicate that loss of extensibility must be related to factors other than oxidation.

\section{Results}

\subsection{Change in material properties over time}

All materials lost extensibility, and this occurred most rapidly in illuminated air, polystyrene being the most extreme with almost complete loss of extensibility within a week (Figure 4). Polyethene (and presumably polystyrene, based on the rate of sample loss through tearing) became unsta-

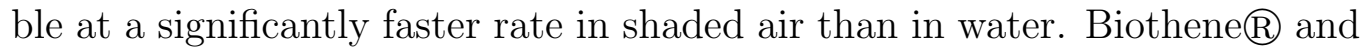
poly(ethylene terephthalate) retained the same stability in shaded air as in water. Replicates of extensibility measurements became progressively fewer (Appendix, Table 3) with increasing exposure duration, as samples became detached from the clamps due to embrittlement.

Molecular constitution also showed changes over time, foremost in polystyrene,

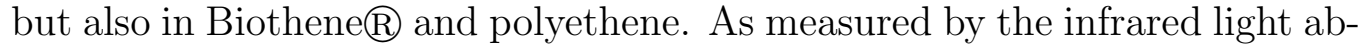
sorption at the specific band, the density of hydroxyl $\left(3450 \mathrm{~cm}^{-1}\right)$, carbonyl $\left(1713 \mathrm{~cm}^{-1}\right)$ and other photo-oxidation products $\left(1100 \mathrm{~cm}^{-1}\right)$ of polystyrene 
Table 3: RELATE statistics from PRIMER v6 showing Spearman's Rho for the correlation of strain and the Carbonyl absorption band value and its significance level based on 9999 random permutations of the values.

\begin{tabular}{llll} 
Environment & Material & Spearman's Rho & Significance $(p)$ \\
\hline Air & Biothene® & 0.614 & $<0.001$ \\
& Polyethene & 0.554 & $<0.001$ \\
\multirow{4}{*}{ Sea water } & Polystyrene & 0.293 & $<0.001$ \\
& Biothene® & 0.034 & 0.2 \\
& Polyethene & 0.006 & 0.5 \\
& Polystyrene & 0.048 & 0.15
\end{tabular}

all increased 2 to 4 times more within 28 days exposure in illuminated air than within 100 days in shaded air. The density increase of these oxidation products was uniform but became slower over time in illuminated air (Figure 5). Changes in the attenuation coefficient of the $1100 \mathrm{~cm}^{-1}$ absorption band between the 21 days and the 28 days cohort were not statistically significant.

\subsection{Difference between environments}

Biothene $囚$, polyethene and polystyrene exhibited considerable oxidation as measured through the carbonyl content in both illuminated and shaded air. No significant change of hydroxyl or carbonyl was recorded in sea water except for a marginal carbonyl increase with polyethene in illuminated sea water (Appendix Table 4).

Polystyrene lost almost all its extensibility after 100 days in both illuminated and shaded sea water, and the uniform loss of extensibility of Biothene® was also faster in both illuminated and shaded sea water than in the control environment. In fact the loss of extensibility was faster in sea water than in air in the beginning. While Biothene® extensbility continued to steadily decrease in air, it did not significantly change between 240 and 600 days in sea water.

The relationship between the molecular constitution and tensile properties was not the same in air as in sea water, as shown by the RELATE statistics in PRIMER-v6 (Table 3). While extensibility and molecular constitution

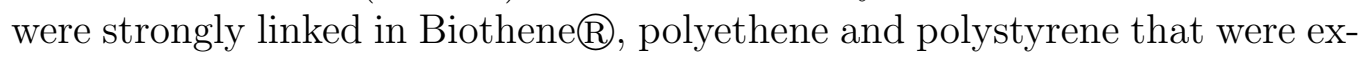
posed in air, there was no such effect in sea water. The analysis showed that in air there was a significant correlation between the number of polymer chain ends (carbonyl groups) and the material's stability as measured by extensibility. In sea water, where material stability was also reduced over time (see Figure 4) but this effect was not correlated with an increase in molecular chain ends. 


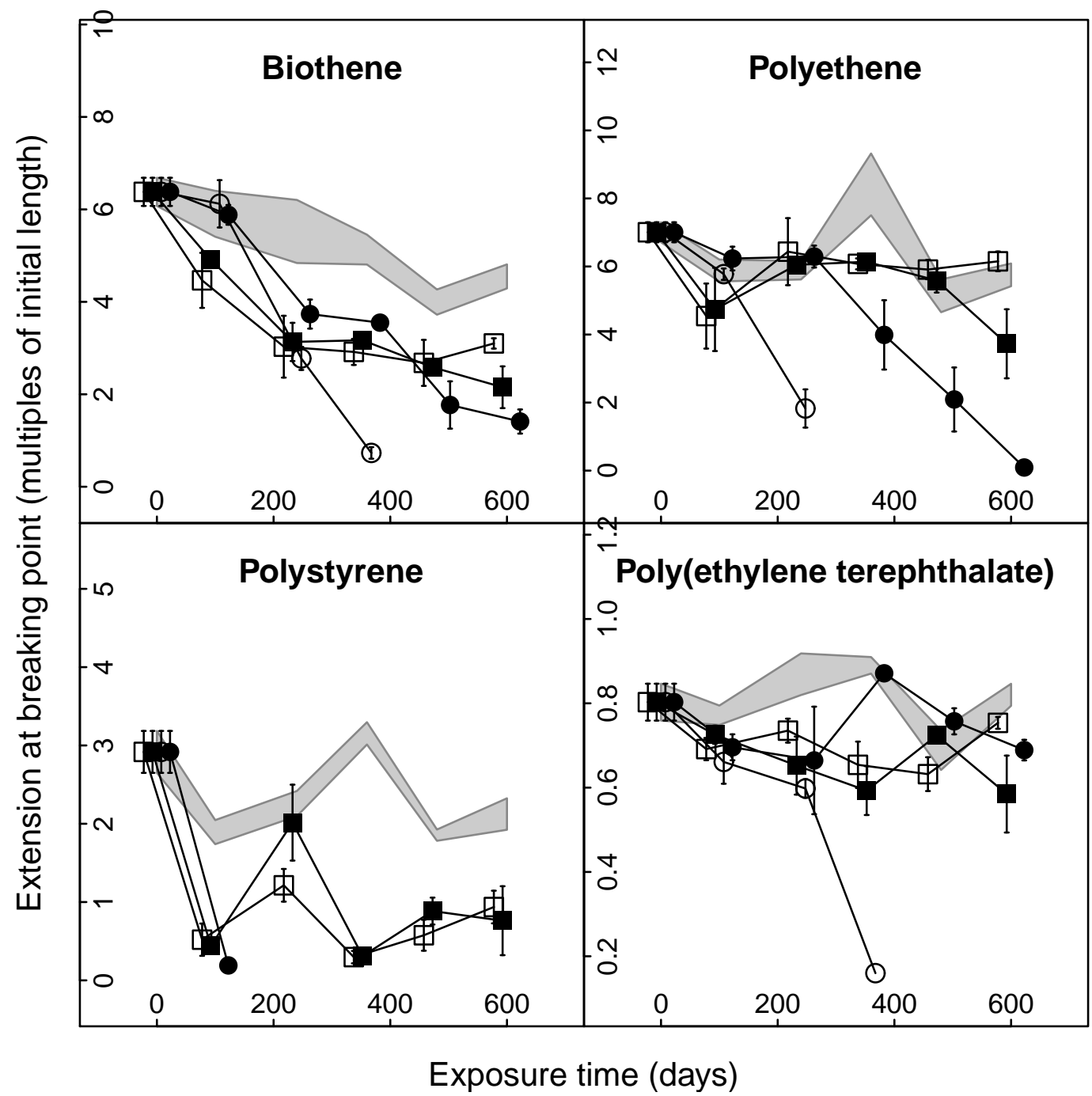

Figure 4: Mean $( \pm s e)$ extensibility at breaking point of all four materials in both environments and both lighting conditions plotted against exposure time (days, $\mathrm{x}$-axis). Open symbols: light; filled symbols: shade; squares: sea water; circles air; shaded area= standard error interval of controls; note that in almost all cases the extensibility was lower in samples exposed in the environment than for the controls. The data series are shifted along the $\mathrm{x}$-axis for clarity. 


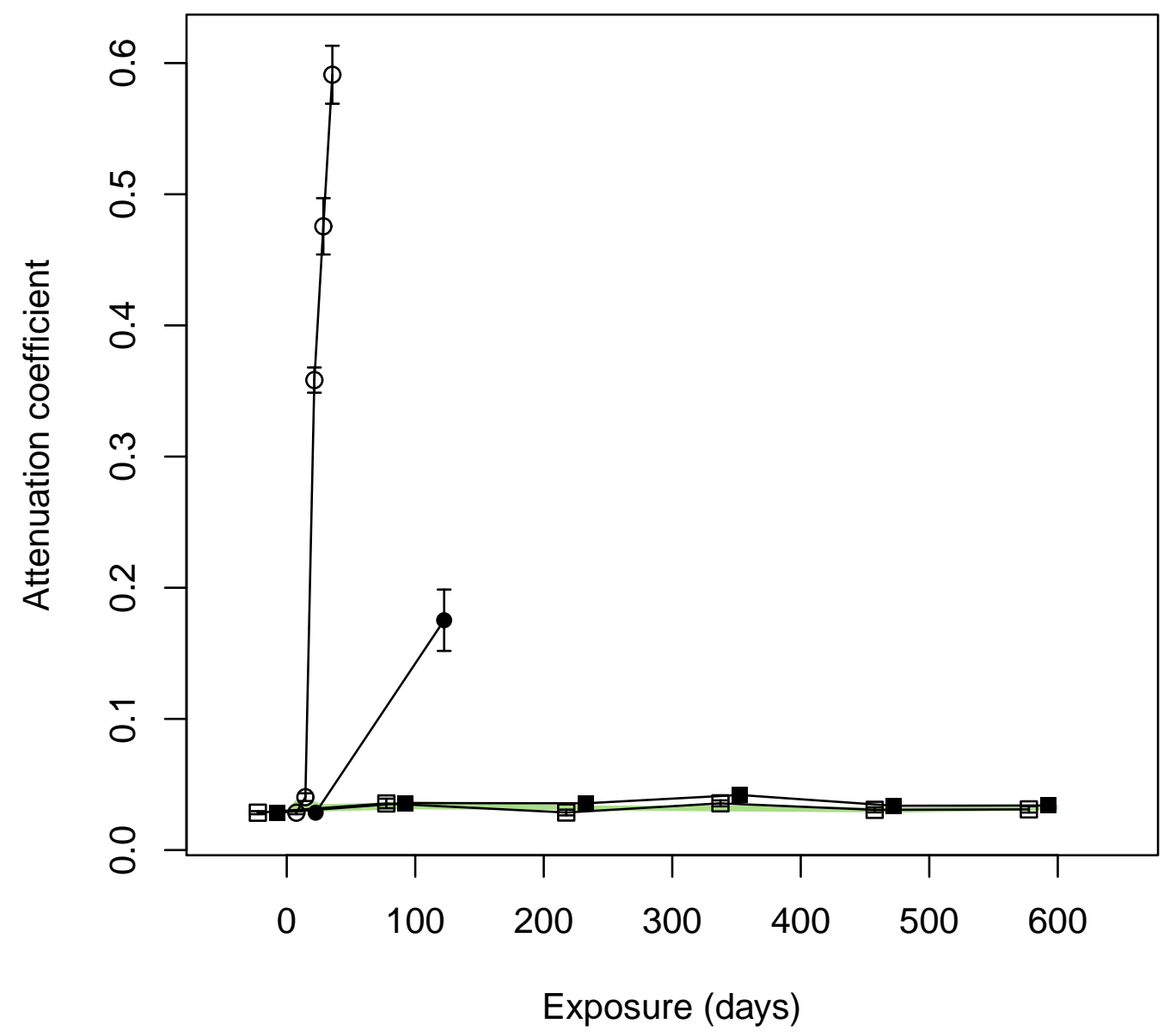

Figure 5: Evolution of the infrared absorption band and standard error of polystyrene at $1713 \mathrm{~cm}^{-1}$ (carbonyl). Circles represent air, squares sea water. Open shapes represent illuminated, solid shapes shaded locations; the green area represents the standard error interval of the control. 


\subsection{Difference in deterioration processes between materials}

Materials differed in changes of extensibility (Appendix Table 3) and carbonyl absorption bands (Appendix Table 4), and there was a significant interaction between deterioration, environment and material. For instance, Biothene $\AA$ deteriorated faster in air compared to sea water than polyethene, while the two materials could not be distinguished on a molecular level as characterised by FTIR.

In terms of tensile properties and molecular constitution, polystyrene deteriorated faster than the other materials (Figure 4). While Biothene® uniformly lost extensibility in all environments including the control, polyethene only started losing extensibility after 240 days exposure in shaded air. Poly(ethylene terephthalate) remained within the variation of the control over the whole duration of the experiment in all environments, except illuminated air, where extensibility appeared to abruptly drop between 240 and 360 days exposure (no statistical analysis as $n=1$ at 360 days).

The substantial increase in the hydroxyl absorption band in illuminated air compared to sea water was clearly different for polystyrene compared to Biothene $\mathbb{B}$ and polyethene. The hydroxyl absorption band did not signifi-

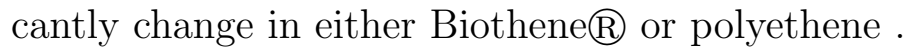

Biothene®, polyethene and polystyrene all underwent a substantial increase in the attenuation coefficient of the carbonyl absorption band in both illuminated and shaded air, but light and exposure duration had a greater effect on the carbonyl content of polysterene than those of Biothene $\mathbb{R}$ and polyethene. Polystyrene forms carbonyl chain ends more rapidly than Biotheneß and polyethene when exposed in illuminated air.

Poly(ethylene terephthalate) most noticeably showed a loss of extensibility in illuminated air, but little effect in other treatments (Appendix Table 3). Materials also differed in how the various treatments affected the rate of deterioration. Light conditions generated a larger effect on polystyrene than on Biothene® or polyethene in samples exposed in air.

\subsection{Effect of light}

Exposure in air - by contrast to sea water - produced a uniform difference of the measured properties between the illuminated and the shaded locations. It also had the greatest difference in irradiance.

The extensibility of polyethene had dropped to twice its original length after 240 days in illuminated air versus six times that in shaded air (Appendix Table 5). The extensibility of Biothene® and poly(ethylene terephthalate) in illuminated air were last measured after 360 days. In shaded air the extensibility of Biothene $\AA$ and polyethene continued to decrease whilst that of poly(ethylene terephthalate) remained constant (Figure 4). 
Changes in the the molecular constitution of polystyrene differed substantially between illuminated and shaded air (Appendix Table 6). The much greater rate of increase of the attenuation coefficient of the carbonyl absorption band was similar to the difference in irradiation between the light and shaded air locations. The evolution of the carbonyl absorption bands of both Biothene $\mathbb{B}$ and polyethene were twice as fast in illuminated as in shaded air. A further difference between illuminated and shaded air in these two materials was the continuity of the change in molecular constitution. In illuminated air the absorption coefficient increased continuously over time, whilst it was highly variable in shaded air.

\section{Discussion}

All materials tested in this study shared one common outcome: they deteriorated much faster in illuminated air than in any of the other environments which reconfirms the findings of Pegram and Andrady (1989) and Andrady et al. (1993c). Extensibility of all materials declined substantially faster in air than in sea water. Polystyrene deteriorated much faster than the other tested materials and showed a high sensitivity to light. In sea water, a factor other than photo oxidation appeared to cause the materials to destabilise, as loss of extensibility was observed, but very little evidence of oxidation was found. However, with the exception of Biothene $\mathbb{R}$ and polystyrene there was no significant loss of extensibility in sea water. Extensibility proved to be a good indicator of material stability. It differentiated between materials and treatments, and illustrated the mechanical consequences of molecular changes.

In terms of importance for marine debris, the faster deterioration rate in air versus sea water means that plastics will begin to deteriorate and consequently to shed microplastics more rapidly on shorelines than when in the sea. Andrady et al. (1993a) suggested that the more rapid deterioration in air was due to higher temperatures and higher UV irradiance, partially due to absence of fouling, although the inherent attenuating properties of seawater themselves were doubtlessly important. An aspect of environmental effect on plastic deterioration that was not addressed in this study was alternation between environments. Future studies could repeat the present experiment but switch the samples between air and sea water, shade and sunlight. In this study deterioration was strongly influenced by the environment. For example, in sea water the materials' tensile properties changed while their molecular constitution remained constant, whilst in air the loss of extensibility was likely related to the presence of oxidised polymer chain ends. Our study was limited by the selection of exemplar materials, which can only be considered 
as representatives of each polymer from a specific manufacturing process. different formulations of each molecule, passing through different processes might respond to environmental exposure in different ways. However, the selected formulations were sourced from carrier bags and packaging film used in the food industry; they are widely used post-consumer materials, and the patterns, similarities and differences highlighted here should stimulate further, more detailed investigations in the future.

Air and sea water differ in more ways than oxygen availability and the intensity of UV radiation, and so a variety of factors may have resulted in the measured differences (i.e. change in molecular constitution and change in tensile properties) between environments. Although individual results for Biothene® and poly(ethylene terephthalate) show an effect of light, this study does not examine the individual factors that contribute to material deterioration, but concentrates upon the difference in deterioration rate between sea water and air. Biothene® deteriorated twice as fast in illuminated air as in sea water, polystyrene about 50 times as fast in air as in sea water. Further work would be needed to establish any environmental consequences of this deterioration; for example the generation of microplastics or the release of additive chemicals. For polyethene and poly(ethylene terephthalate) no measurable deterioration occurred in sea water over the course of the study.

While Biothene®, polyethene and poly(ethylene terephthalate) remained stable for 3 to 6 months even in illuminated air, polystyrene lost almost all of its extensibility in a matter of days. Polystyrene has a strong reaction to UV radiation, because it has tertiary bonds that accelerate oxidation propagation and hinder termination (Amin and Scott, 1974). This means that a piece of polystyrene that is washed up on a beach possibly after years of being in the ocean could become extremely brittle in a relatively short period of time, and very little mechanical stress would be needed to cause it to then shed microplastics. Indeed, previous studies have reported that plastics in the marine environment deteriorate primarily on beaches as the temperature, friction and UV irradiance are higher than in water (Gregory and Andrady, 2003). This study therefore provides a semi-quantitative illustration of potential deterioration of 4 types of plastic on beaches (our samples in air) versus in seawater, indicating a difference of two orders of magnitude for polystyrene.

Biothene®, polyethene and poly(ethylene terepthalate) did not deteriorate as rapidly as polystyrene in illuminated air, but did so at an accelerated rate compared to in sea water. Items that are made from these materials that are washed ashore are also likely to produce microplastics over a longer period of time. It is therefore possible that a large portion of theh microplastics now 
present in the ocean may have been formed by fragmentation out of water, for instance on the shoreline when washed up or before entering the ocean on a source beach, supporting a hypothesis that the stock of these materials currently in the sea represents a potential future source of microplastics for years to come. Plastics may, of course, alternate between seawater and areas further inland than the shoreline, which would call for an adjustment in any estimate of proportion of time spent on land versus sea water. However, this would not affect the outcome of this study or the conclusion regarding the role of sea water slowing down deterioration compared to air.

Previous studies attributed lower rates of extensibility loss in sea water than in air to lower temperatures (Pegram and Andrady, 1989) and to biofouling (Andrady et al., 1993a) which inhibit oxidation . This study suggests that any loss of structural integrity in sea water occurs for reasons other than oxidation. Very little evidence of oxidation of Biothene®, polyethene and polystyrene was observed in sea water. Polystyrene and Biothene®, which exhibited substantial loss of extensibility in air likely linked to oxidation, also showed less loss of extensibility in sea water, but more than the indicated levels of oxidation could account for, based on the observed relationship between oxidation and extensibility loss in air. Biofouling has been listed as a factor promoting loss of structural integrity (Andrady et al., 1993a) especially on materials with starch additives. Neither material with significant loss of extensibility in sea water had such additives, but substantial biofouling was observed and may potentially contribute to material deterioration in other ways. Some possible effects of biofouling on material stability are increasing drag and chronic shear stress in floating debris or through some other biochemical effect of fouling.

This study challenges the environmental relevance of plastics with enhanced degradability, as Biothene $\mathbb{R}$ deteriorated in much the same timescale as conventional polyethene. Concerns have been raised over the effectiveness of metal ion additives to improve degradability (Osawa, 1988); some of these materials may simply present a faster way for microplastics to form from larger debris. However, microplastics aside, in the current experiment

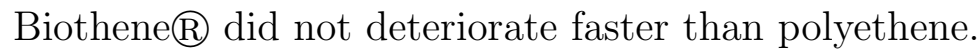

\section{Conclusion}

Out of the three widely used conventional materials polyethene, polystyrene and poly(ethylene terephthalate) and one example of a 'degradable plastic'

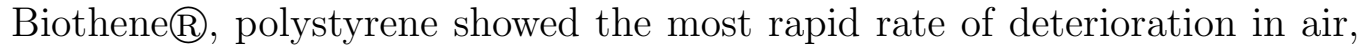
especially when directly exposed to sunlight. Polystyrene is widely used in packaging (as expanded polystyrene cushioning or seal-wrapping for food 
items); therefore, it typically has a short service life and is likely to end up in the environment more frequently than many other polymers. It is crucial to recognise that while the polystyrene deteriorated rapidly the study did not quantify biodegradability for any of the materials, and the most likely consequence of the deterioration of polystyrene is the formation of microplastic particles. It is, therefore, advisable to further quantify the relative importance of polystyrene as a source of microplastics in the environment.

The experiment showed that the deterioration of all four tested materials occurred more quickly in air than in $1 \mathrm{~m}$ of sea water. This was evidenced by measurements of loss of material, loss of extensibility and changes in molecular composition within a maximum exposure period of six hundred days. The molecular composition of the samples exposed in sea water did not change, and it is possible that water reduces photo-oxidation regardless of the UV radiation through a low concentration of dissolved oxygen. The marine environment where plastics are most likely to deteriorate the fastest is the intertidal. Marine debris may drift in the sea, cooled by water, shielded from UV radiation and exposed to little oxygen. As a consequence plastic items may remain intact at sea for many years. It is therefore likely that intertidal habitats play an important role in the formation of microplastics through deterioration in the marine environment. Beach surveys may provide valuable information on the generation of microplastics, and beach clean-ups may be an effective way to help reduce the generation of microplastics in marine environments.

\section{Acknowledgements}

This study was funded by the Theodor Engelmann Stiftung, Erika Klemm Roniger, Marianne Klemm and the Biber family. It was conducted with the invaluable assistance of Josh Bam, Alison Biber, Chris Booker, Roger Crook, Roger Haslam, Rebecca Harris, Danni Hodgson, the friendly staff at Mayflower Marina, Terry Richards, Fabian Rudin, Richard Ticehurst, Andrew Tonkin, Ann Torr, Michael Whelan and Michael Whiffen.

Allen, N., Fatinikun, K., Henman, T., 1983. Thermal and photochemical oxidation of polypropylene. influence of residual catalyst levels in unstabilised diluent and gas phase polymers. European Polymer Journal 19 (7), $551-554$.

Amin, M., Scott, G., 1974. Photo-initiated oxidation of polyethylene effect of photo-sensitizers. European Polymer Journal 10 (11), 1019-1028. 
Anderson, M. J., Gorley, R. N., Clarke, K. R., 2008. PERMANOVA+ for PRIMER: Guide to Software and Statistical Methods. PRIMER-E, Plymouth.

Andrady, A., 2003. Plastics in the environment. In: Andrady, A. (Ed.), Plastics and the environment. John Wiley \& Sons, Inc., Hoboken, p. 762.

Andrady, A., Pegram, J., Tropsha, Y., 1993a. Changes in carbonyl index and average molecular weight on embrittlement of enhanced photo-degradable polyethylene. Journal of Polymers and the Environment 1 (3), 171-179.

Andrady, A. L., Pegram, J. E., Nakatsuka, S., 1993b. Studies on enhanced degradable plastics: 1 . the variability in outdoor lifetimes of enhanced photodegradable polyethylenes. Journal of Environmental Polymer Degradation 1 (1), 31-43.

Andrady, A. L., Pegram, J. E., Song, Y., 1993c. Studies on enhanced degradable plastics. ii. weathering of enhanced photodegradable polyethylenes under marine and freshwater floating exposure. Journal of Environmental Polymer Degradation 1 (2), 117-126.

Barnes, D. A., 2005. Remote islands reveal rapid rise of southern hemisphere sea debris. Science World Journal 5, 915-921.

Bond, A. L., Provencher, J. F., Daoust, P.-Y., Lucas, Z. N., 2014. Plastic ingestion by fulmars and shearwaters at sable island, nova scotia, canada. Marine Pollution Bulletin 87 (1), 68-75.

Brandon, J., Goldstein, M., Ohman, M. D., 2016. Long-term aging and degradation of microplastic particles: Comparing in situ oceanic and experimental weathering patterns. Marine Pollution Bulleting 110 (1), 299-308.

British Standards Institution, 1 1996. Plastics - determination of tensile properties. Tech. rep.

Browne, M., Dissanayake, A., Galloway, T., Lowe, D., Thompson, R., 2008. Ingested microscopic plastic translocates to the circulatory system of the mussel, mytilus edulis (1.). Environmental Science and Technology 42 (13), 5026-5031.

Carrasco, F., Pags, P., Pascual, S., Colom, X., 2001. Artificial aging of highdensity polyethylene by ultraviolet irradiation. European Polymer Journal 37 (7), 1457-1464. 
Clarke, K. R., Gorley, R. N., 2006. PRIMER v6: User Manual/Tutorial. PRIMER-E, Plymouth.

Cole, M., Webb, H., Lindeque, P., Fileman, E., Halsband, C., Galloway, T., 3 2014. Isolation of microplastics in biota-rich seawater samples and marine organisms. Scientific Reports 4 (4528), 1-8.

Colton, J., Knapp, F., Burns, B., 1974. Plastic particles in surface waters of the northwestern atlantic. Science 185 (4150), 491-497.

Copinet, A., Bertrand, C., Govindin, S., Coma, V., Couturier, Y., 2004. Effects of ultraviolet light $(315 \mathrm{~nm})$, temperature and relative humidity on the degradation of polylactic acid plastic films. Chemosphere 55 (5), $763-773$.

Gardette, J.-L., Mailhot, B., Lemaire, J., 1995. Photooxidation mechanisms of styrenic polymers. Polymer Degradation and Stability 48 (3), 457-470.

Goldstein, M. C., Goodwin, D. S., 2013. Gooseneck barnacles (Lepas spp.) ingest microplastic debris in the north pacific subtropical gyre. PeerJ 1, e184.

Gregory, M., 1978. Accumulation and distribution of virgin plastic granules on new zealand beaches. New Zealand Journal of Marine and Freshwater Research 12 (4), 339-414.

Gregory, M., Andrady, A., 2003. Plastic in the marine environment. In: Andrady, A. (Ed.), Plastics and the environment. John Wiley \& Sons, Inc., Hoboken, pp. 379-401.

Hamid, S., Prichard, W., 1991. Mathematical modelling of weather induced degradation of polymer properties. Journal of Applied Polymer Science 43 (4), 651-678.

ISO/DIS, 2009. 10640: Plastics - method of assessing accelerated photoageing by ftir and uv/visible spectrometry. Tech. rep.

Israeli, Y., Lacoste, J., Lemaire, J., Singh, R., Sivaram, S., 1994. Photoand thermoinitiated oxidation of high-impact polystyrene. i. characterization by ft-ir spectroscopy. Journal of Polymer Science: Part A: Polymer Chemistry 32 (3), 485-493.

Klemchuck, P., 1990. Degradable plastics: A critical review. Polymer Degradation and Stability 27 (2), 183-202. 
Kukulka, T., Proskurowski, G., Mort-Ferguson, S., Meyer, D. W., Law, K. L., 2012. The effect of wind mixing on the vertical distribution of buoyant plastic debris. Geophysical Research Letters 39 (L07601), 1-6.

Kuzina, S., Mikhailov, A., 2001. Photo-oxidation of polymers-4. the dual mechanism of polystyrene photo-oxidation: a hydroperoxide and a photochain one. European Polymer Journal 37 (11), 2319-2325.

Lee, H., Shim, W., Kwon, J.-H., 2014. Sorption capacity of plastic debris for hydrophobic organic chemicals. Science of the Total Environment 470, $1545-1552$.

Lucas, Z., 1992. Monitoring persistent litter in the marine environment on sable island, nova scotia. Marine Pollution Bulletin 24 (4), 192-199.

Marek, A., Kápralková, L., Schimdt, P., Pfleger, J., Humliček, J., Pospíšil, J., 2006. Polymer spatial resolution of degradation in stabilized poolystyrene and polypropylene plaques exposed to accelerated photo-degradation or heat aging. Polymer Degradation and Stability 91 (3), 444-458.

Nagai, Y., Ogawa, T., Nishimoto, Y., Ohishi, F., 1999. Analysis of weathering of a thermoplastic polyester elastomer ii. factors affecting weathering of a polyether-polyester elastomer. Polymer Degradation and Stability 65 (2), $217-224$.

Nakayama, Y., Takahashi, K., Sasamoto, T., 1996. Esca analysis of photodegraded poly(ethylene terephthalate) film utilizing gas chemical modification. Surface and Interface Analysis 24 (10), 711-717.

Obadal, M., Čermák, R., Raab, M., Verney, V., Commereuc, S., Frä̈sse, F., 2006. Study on photodegradation of injection moulded (beta)polypropylene. Polymer Degradation and Stability 91 (3), 459-463.

O'Brine, T., Thompson, R., 12 2010. Degradation of plastic carrier bags in the marine environment. Marine Pollution Bulletin 60 (12), 2279-2283.

Osawa, Z., 1988. Role of metals and metal-deactivators in polymer degradation. Polymer Degradation and Stability 20 (3), 203-236.

Pegram, Andrady, J. A., 1989. Outdoor weathering of selected polymeric materials under marine exposure conditions. Polymer Degradation and Stability 26 (4), 333-345.

PlasticsEurope, 2012. Plastics the facts 2012. Tech. rep. 
Ranby, B., 1989. Photodegradation and photo-oxidation of synthetic polymers. Journal of Analytical and Applied Pyrolysis 15, 237-247.

Sawai, D., Nagai, K., Kubota, M., Ohama, T., Kanamoto, T., 2006. Maximum tensile properties of oriented polyethylene, achieved by uniaxial drawing of solution-grown crystal mats: Effects of molecular weight and molecular weight distribution. Journal of Polymer Science:Part B: Polymer Physics 44 (1), 153-161.

Singh, B., Sharma, N., 2008. Mechanistic implications of plastic degradation. Polymer Degradation and Stability 93 (3), 561-584.

Song, Y. E., Andrady, A. L., 1991. Fouling of floating plastic debris under biscayne bay exposure conditions. Marine Pollution Bulleting 22 (12), 608613.

Teuten, E., Rowland, S., Galloway, T., Thompson, R., 11 2007. Potential for plastics to transport hydrophobic contaminants. Environmental Science and Technology 41 (22), 7759-7764.

Thompson, R., Olsen, Y., Mitchell, R., Davis, A., Rowland, S., John, A. G., McGonigle, D., Russel, A., 5 2004. Lost at sea: Where is all the plastic? Science 304 (5672), 838.

van Cauwenberghe, L., Michiel, C., Vandegehuchte, M., Janssen, C., 2 2012. Occurrence of microplastics in mussels (Mytilus edulis) and lugworms (Arenicola marina) collected along the french-belgian-dutch coast. In: VLIZ Special Publications. Vol. 55. pp. 87-87. 